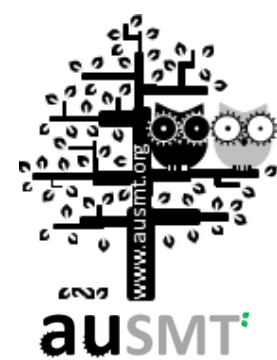

\title{
Trends of System Management Software Development in the Context of Data Center Management Automation
}

\section{Yi-Ling Wei *}

Industrial Economics and Knowledge Center (IEK), Industrial Technology Research Institute (ITRI), Taiwan

(Received 29 April 2013; Published on line 1 June 2013)

*Corresponding author: yyhuang@itri.org.tw

DOI: 10.5875/ausmt.v3i2.206

Abstract: In the past, data centers were designed as independent hardware system and management software. With the shift from cloud-computing architecture to integrated system management and with continuing requests for lower costs, data centers are developing in the direction of automated management, which has made data center system management software increasingly important. System management software mainly provides functions like environmental monitoring, automatic control, energy monitoring, which can help data centers with integrated management and maintenance conveniently.

Keywords: Cloud data center; System Management Software; Virtualization; DCIM (Datacenter Infrastructure Management)

\section{Introduction}

Since the 1980s, data center architecture, whether Mainframe-based or gradually modern X86, has been developed with focus on the efficiency of data processing as well as the constant lowering of cost. In recent years, with the rise of cloud data center architecture, data centers are prone to use a good number of low-cost hardware combined with data management software to achieve the purpose of high efficiency and low cost.

Cloud data center architecture, different from the construction logic of data centers in the past in which hardware systems were respectively separated, adopts unified management instead. By means of favorable distributed replication model, big data can be instantly processed so as to achieve high efficiency. Furthermore, instead of using high-spec fault-tolerant hardware, the errors of hardware are deemed normal status and tasks are assigned through system management software. The above-mentioned update in architecture and data center calculation logic attributes to remarkable advantages of the new generation of cloud data centers in terms of cost and efficiency. It's estimated that in 10 years, with the rise of various consumer cloud services, cloud data center architecture will gradually become the mainstream architecture among global data centers.

\section{The Definition of System Management Software for Cloud Data Centers}

According to studies of IDC, an international research institution, system management software for data centers refers to the software that provides planning, management and optimization functions as well as the service that manages space, energy and cooling system. This type of software and service usually focuses on the relations between non-ICT software architecture and IT systems. As a result, the operation of service or software will not only involve the management 
of a single IT hardware or non-ICT hardware architecture, but will consider the data centers as a whole.

And Gartner, a market research institution, defines system management software for data centers as tools that not only monitor, measure, manage and control the operation of a variety of information hardware (server, storage, networking) and non-ICT hardware (power, cooling), but also manages energy consumption. This kind of software can realize the following functions: 1) help improve the operational efficiency of information hardware and other facilities within the data center, 2) help evaluate the optimized designs while the quantity of hardware in the data center is increasing, 3) integrate the management of IT hardware and non-ICT hardware, and 4) control energy consumption of data centers by use of management tools so as to be in accordance with related laws and regulations.

In summarizing the definitions of system management software for data centers by the international research institutions above, some common points are extracted as follows: 1) the scope of system management software includes IT hardware and non-ICT facilities, and 2) the software's main functions are to help monitor, manage and plan the operation of all hardware in data centers as well as to help data centers develop toward the goals of automation, optimization and cost-effectiveness.

\section{Functions of System Management Software for Data Centers}

In order to save the manpower management cost by improving system management software efficiency, enterprises in recent years have been actively introducing all kinds of automated management software and system monitoring software, so they can substantially reduce manpower cost by integrating management software with the colossal hardware system.

As the form of data center has gradually transformed from the single hardware architecture to a highly integrated and modularized one, the issue of energy management has become more and more important, especially in the new form of data centers. Hardware cost has been significantly reduced due to modularization; however, energy cost has considerably increased because of the integrated architecture and large-scale hardware, which has become the most

Yi-Ling Wei is a researcher in the Electronics \& System Research Division at Industrial Economics and the Knowledge Center. Her Research Topics Focus on Cloud Computing, Data Center, Mobile Applications, and so on. expensive part in data centers. Consequently, how to analyze and control changes of variable cost by means of space planning and software monitoring tools becomes a priority in the development of data centers.

Therefore, in order to assist enterprises in managing data centers with dynamic configurations, system management software for data centers mainly provides functions as follows:

\section{- Environmental monitoring:}

Environmental changes related to factors like safety, temperature and even disaster prevention have significant impact on the operation of data centers. With the trend of unmanned management, the functions of monitoring environmental changes of data centers in real time and providing immediate feedback to control system for automatic response to emergency would become very important. For instance, when a sudden surge in temperature of a specific server rack is detected, environmental monitoring function can report the temperature change to the system so that the automatic control can lower the temperature or cut off power.

\section{- Automatic control:}

It lies in assisting the automatic control of equipment within the data center. Once orders are given through external management systems, the settings of equipment or IT environment can be altered automatically, such as the increase or decrease of a single server's temperature or energy without the interference of manpower.

\section{- Energy management:}

Energy monitoring function is currently a focus of DCIM software development, mainly because energy cost, which is getting higher day by day, accounts for a high percentage in variable cost. For this reason, energy control shall be the most effective way to cost-cut. Apart from power usage monitoring and analysis, energy control also includes the control of energy usage effectiveness, such as PUE (Power Usage effectiveness) reporting function. PUE is an index used to assess what proportion of power is consumed by IT equipment in a data center. It's a fairly important indicator in the assessment of data center efficiency at present that makes recording and comparing energy usage efficiency more conveniently.

\section{- Integrated management tools:}

Currently, system management software is largely based on integrated management of information hardware and non-ICT hardware architecture. 
Therefore, how to integrate previous system management software into management system seamlessly under the existing operational environment becomes a vital topic. This kind of tool mainly provides necessary information and data to other management software to achieve integrated system management. For example, it can provide energy and temperature related information to virtualization management software.

\section{- Situational analysis:}

Situational analysis is very helpful to cloud data centers. Nowadays, the new type of data centers is constructed phase by phase and with increased number of equipment according to specific demands, totally different from the one-off plan and construction mode. Therefore, situational analysis can help data center administrators to quickly analyze ways to allocate newly bought equipments in terms of the condition of space, floor load-bearing and energy supply, so as to optimize operational configuration in data centers.

\section{The Development Trends of System Management Software for Data Centers}

Data centers are developing continuously towards dynamic configuration optimization, modularized intelligent building and high energy efficiency. According to the data center cost analysis in recent years, the cost of energy and cooling system is far higher than that of initial data center construction. This finding shifted the focus of system management software development to the reduction of variable cost like energy and cooling system cost.

Owing to the trends mentioned above, in the process of system management software design, product functions are adjusted according to the design architecture of dynamic IT configurations, with the purpose of integrating the best system configurations in conformity with current IT demands, so that optimized energy usage efficiency can be achieved.

And therefore, the future system management software will be based on the integrated management of information hardware and non-ICT equipment. Under the influence of growing demand for lower cost, functions concerning energy efficiency monitoring and management will become a main development direction in the near future. Thus functions including environmental monitoring, energy management and integrated management tools will be the priorities for system management software development in the near future.
Over time, automatic control function and situational analysis will become the follow-up development focus as a result of the demand for unmanned management and dynamic IT configuration optimization.

\section{Conclusion}

The above-mentioned development of system management software for data centers will become increasingly crucial as data centers' construction logic is directed to dynamic configuration mode. And therefore, when designing information hardware products in the future, we have to consider enterprises' demands for real-time control of operational environment, status and automatic control in data center. Furthermore, to meet the requirements of data center operators, we even have to consider integrating some system management software into hardware so as to be sold together. In particular, how to help enterprises quickly reduce variable cost (e.g. energy cost) in the short term and how to help lower management cost and complexity for data centers will be main issues that related manufacturers should make constant efforts to deal with.

\section{References}

[1] Data center solution for delta electronics, [Online]. Available: http://www.delta.com.tw/index.asp

[2] K. Broderick, "Worldwide datacenter infrastructure management 2011-2015 forecast," IDC, 2012.

[3] M. J. Turner, "Worldwide cloud systems management software 2011 vendor strategies," IDC, 2011.

[4] H. Cecci and J. E. Pultz, "Research roundup for data center infrastructure management tools, 1Q13," Gartner, 2013.

[5] F. De Silva, J. E. Pultz, and A. Adams, "Market trends: Total addressable dcim market will reach \$1.7 billion by 2016," Gartner, 2012.

[6] A. Adams and J. E. Pultz, "User survey analysis: What enterprise users are saying about their datacenter infrastructure management plans," Gartner, 2012. 